\title{
Hypothalamic pro-opiomelanocortin gene delivery ameliorates obesity and glucose intolerance in aged rats
}

Received: 21 March 2005 / Accepted: 20 June 2005 / Published online: 5 October 2005

(C) Springer-Verlag 2005

\begin{abstract}
Aims/hypothesis: Age-related obesity is associated with impaired hypothalamic pro-opiomelanocortin (Pomc) gene expression. We assessed whether overproduction of POMC in the hypothalamus ameliorates age-related obesity in rats. Methods: Recombinant adeno-associated virus (rAAV) encoding Pomc (rAAV-Pomc) or control vector was delivered bilaterally into the basomedial hypothalamus of aged obese rats with coordinates targeting the arcuate nucleus. Energy balance, glucose metabolism, brown adipose tissue thermogenesis and mRNA levels of hypothalamic neuropeptides and melanocortin receptors were assessed. Results: Forty-two days after Pomc gene delivery, hypothalamic Pomc expression increased 12-fold while agouti-related protein and neuropeptide Y mRNA levels remained unchanged. Using a punch technique, we detected the highest Pomc RNA level in the arcuate nucleus. Pomc overexpression reduced food consumption from day 10 after vector injection, but this anorexic effect abated by day 30 . In contrast, there was a steady decrease in body weight without apparent attenuation. Pomc gene delivery decreased visceral adiposity and induced uncoupling protein 1 in brown adipose tissue in aged rats. Serum NEFA and triglyceride levels were also diminished by rAAV-Pomc treatment. Improved glucose metabolism and insulin sensitivity were observed on day 36 but not day 20 after Pomc gene delivery. The expression of hypothalamic melanocortin 3 and 4 receptor decreased by $17 \%$ and $25 \%$, respectively in rAAV-Pomc rats. Conclusions/ interpretation: This study demonstrates that targeted Pomc gene therapy in the hypothalamus reduces body weight and
\end{abstract}

G. Li · Y. Zhang · J. T. Wilsey · P. J. Scarpace

Department of Pharmacology and Therapeutics, University of Florida College of Medicine,

Gainesville, FL, USA

Y. Zhang · J. T. Wilsey $\cdot$ P. J. Scarpace $(\bowtie)$

Geriatric Research, Education and Clinical Center,

Malcom Randall Veterans Affairs Medical Center,

Gainesville, FL 32608-1197, USA

e-mail: scarpace@ufl.edu

Tel.: +1-352-3746114

Fax: +1-352-3746142 visceral adiposity, and improves glucose and fat metabolism in aged obese rats. Thus long-term activation of the central melanocortin system may be a viable strategy to combat age-related obesity and diabetes.

Keywords AAV · Age-related obesity · Diabetes . Gene therapy $\cdot$ Pomc

Abbreviations AAV: adeno-associated virus - Agrp: agouti-related protein $\cdot$ F344/BN: Fischer 344-Brown Norway rats - IPGTT: intraperitoneal glucose tolerance test $\cdot M c 3 r$ : melanocortin 3 receptor $\cdot M c 4 r$ : melanocortin 4 receptor $\cdot \alpha$-MSH: $\alpha$-melanocyte stimulating hormone $\cdot$ MTII: melanotan II - Npy: neuropeptide Y P Pomc: pro-opiomelanocortin $\cdot$ rAAV: recombinant adenoassociated virus - rAAV-Pomc: rAAV encoding Pomc . UCP1: uncoupling protein 1

\section{Introduction}

Melanocortins are bioactive peptides derived from a common prehormone, pro-opiomelanocortin (POMC); the central melanocortin system plays a critical role in the regulation of energy balance and glucose metabolism [16]. Reduced expression of hypothalamic Pomc is associated with obesity syndromes caused by: (1) mutations in any of several genes, including leptin receptor $[7,8]$, tubby [9] and Nhlh2 [10]; (2) hypothalamic damage [11]; and (3), perhaps most commonly, by ageing [12]. Reduced hypothalamic Pomc mRNA may be one contributor to the obese phenotypes in these models because mutations in the Pomc gene cause obesity in mice [13] and humans [14]. However, there is still little evidence that normalisation of central POMC tone can reverse obese phenotypes. The present study aimed to address this question using an age-related obese rat model.

A common form of obesity, age-related obesity, is characterised by a progressive increase in body weight and visceral adiposity. This increase in body weight with age is a major risk factor for insulin resistance, diabetes and ath- 
erosclerotic cardiovascular disease [15]. Aged mice and rats are associated with reduced hypothalamic Pomc expression [12], and the induction of Pomc by exogenous leptin is impaired with ageing [16]. The male F1 hybrid of Fischer 344-Brown Norway rats (F344/BN) is a useful model for age-related obesity because these rats have a relatively long lifespan and gain a substantial amount of body weight and fat mass with ageing [17]. F344/BN rats also display age-associated impairments in glucose metabolism and insulin responsiveness [18]. Although chronic pharmacological treatment of melanocortin agonists in rodents, including aged rats, reduces food intake and increases energy expenditure, its effectiveness is limited by the rapid tachyphylaxis of the melanocortin responses [1921]. On the other hand, we have demonstrated previously that Pomc gene delivery mediated by recombinant adenoassociated virus (rAAV) elicited a sustained anorexic response (up to 38 days) in obese Zucker rats with defective leptin receptors [22]. Using the F344/BN rat as a model of age-related obesity, we sought to examine whether overproduction of POMC in the hypothalamus could reverse the metabolic impairments associated with age, by reducing body fat and weight while improving glucose metabolism. In addition, we hypothesised that long-term targeted overexpression of Pomc in the hypothalamus would result in a prolonged anorexic response compared with the pharmacological administration of melanocortin agonists.

The advantages of using rAAV to obtain long-term transgene expression include site-specific integration within a defined region of human chromosome 19 , the ability to transduce postmitotic tissues efficiently, and a lack of pathogenicity and immunogenicity [23]. We have demonstrated previously that a serotype $2 \mathrm{rAAV}$ vector encoding $P$ omc reduces visceral adiposity and improves insulin sensitivity in genetically obese Zucker rats [22]. A new serotype rAAV, rAAV type 5, has been developed recently and shown to be more efficient in transducing select tissues in vivo $[24,25]$. In the present study, we used a serotype type 5 rAAV vector encoding murine Pomc (rAAV-Pomc) to assess the long-term consequences of Pomc gene delivery on energy balance, glucose metabolism, brown adipose tissue thermogenesis and mRNA levels of hypothalamic neuropeptides and melanocortin receptors in aged obese F344/ $\mathrm{BN}$ rats.

\section{Materials and methods}

\section{Construction of rAAV vector plasmids}

pTR-Pomc encodes the full-length 935-bp murine Pomc cDNA [26] under the control of the hybrid cytomegalovirus immediate early enhancer/chicken $\beta$-actin promoter [27]. The woodchuck hepatitis virus post-transcriptional regulatory element is placed downstream of the Pomc transgene to enhance its expression [28] (Fig. 1). The control plasmid, termed pTR-control, is similar to pTR-Pomc except for the incorporation of the cDNA encoding an enhanced form of green fluorescent protein instead of Pomc cDNA. The control vector has been described previously [29].

\section{Packaging of rAAV vectors}

The plasmids pTR-Pomc and pTR-control were packaged in serotype 5 rAAV capsids. Serotype 5 was produced by the process known as 'pseudotyping', by using the helper plasmid pXYZ5 [30], containing the rAAV 5 capsid genes. Vectors were packaged, purified, concentrated and titred as described [30]. The titres for the rAAV-Pomc and rAAVcontrol vectors used in this study were $2.51 \times 10^{13}$ physical particles $/ \mathrm{ml}$.

\section{Animals}

Male F344/BN rats aged 22 months were obtained from Harlan Sprague-Dawley (Indianapolis, IN, USA) under contract with the National Institute on Aging. Animals were cared for in accordance with the principles of the NIH Guide to the Care and Use of Experimental Animals. Rats were housed individually with a 12:12 h light:dark cycle (lights on 0700 to 1900 hours). Free access was provided to standard Purina 5001 rodent diet and water.

\section{Administration of $\mathrm{rAAV}$ vector}

Under anaesthesia induced by $6 \mathrm{mg} / \mathrm{kg}$ xylazine (Phoenix Pharmaceutical, St Joseph, MO, USA) and $60 \mathrm{mg} / \mathrm{kg} \mathrm{ke}-$ tamine (Monarch Pharmaceuticals, Bristol, TN, USA), rats were bilaterally administered rAAV-Pomc $(n=12)$ or rAAV-
Fig. 1 Schematic presentation of the rAAV-Pomc construct. TR $\mathrm{AAV}$ terminal repeat sequence; $C M V$ ie enhancer cytomegalovirus immediate-early enhancer; WPRE woodchuck hepatitis virus post-transcriptional regulatory element; $b G H \operatorname{poly}(A)$ bovine growth hormone polyadenylation signal

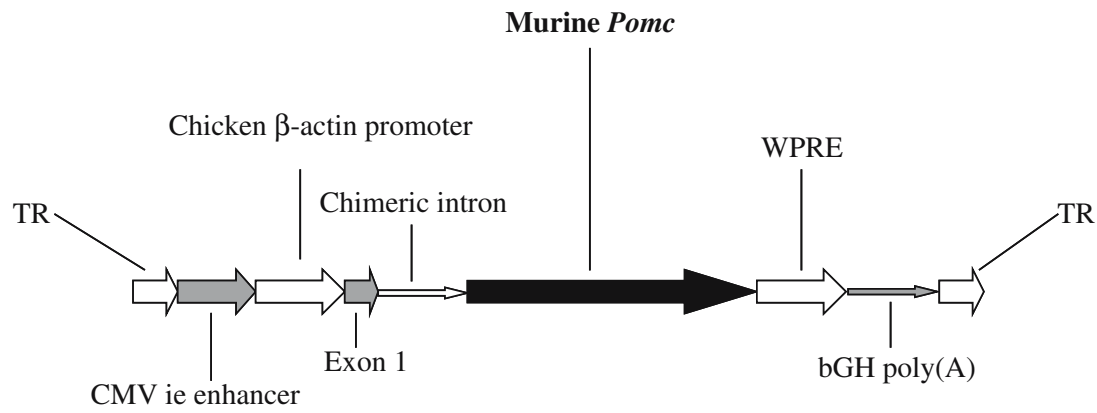


control $(n=12), 2.51 \times 10^{10}$ particles/injection in $1 \mu$, or artificial cerebrospinal fluid (surgical sham control, $n=5,1 \mu \mathrm{l}$ per injection) into the basomedial hypothalamus with coordinates targeting the arcuate nucleus, as described previously [22]. Using an UltraMicropump II system (World Precision Instruments, Sarasota, FL, USA), a 1- $\mu$ l volume of virus stocks was delivered over 5 min to each site. The needles remained in place at the injection site for a further $5 \mathrm{~min}$. At the time of surgery, rats were injected with the analgesic Buprenex (buprenorphine hydrochloride, $0.05 \mathrm{mg} / \mathrm{kg}$; Reckitt and Colman, Richmond, VA, USA).

Intraperitoneal glucose tolerance test

An intraperitoneal glucose tolerance test (IPGTT) was performed on days 20 and 36 after vector administration. Rats were fasted overnight and injected intraperitoneally with glucose ( $2 \mathrm{~g} / \mathrm{kg}$ body weight) at 10.00 hours. blood was taken from the tail vein immediately before glucose injection and 15, 30, 60 and $120 \mathrm{~min}$ after injection. Blood glucose was measured by One Touch SureStep glucose meter (LifeScan, Milpitas, CA, USA). Plasma insulin concentrations during IPGTT were also measured by a rat insulin ELISA kit (Linco Research, St Charles, MO, USA).

Tissue collection and preparation

Rats were killed by cervical dislocation under $100 \mathrm{mg} / \mathrm{kg}$ pentobarbital anaesthesia at days 22 and 42 . Cardiac blood, hypothalamus, brown adipose tissue and perirenal, retroperitoneal and epididymal white adipose tissues were obtained as described previously [22]. For dissection of hypothalamic nuclei, two groups of rats $(n=6$ for rAAVPomc and rAAV-control, respectively) were killed and brains were rapidly excised, chilled on ice-cold saline and sliced using a Stoelting tissue slicer. Brains were sectioned at $0,-2$ and $-5.0 \mathrm{~mm}$ relative to the anterior commissure, corresponding to the brain atlas of Paxinos and Watson [31]. The paraventricular nucleus was removed from the slices taken at 0 and $-2 \mathrm{~mm}$, and the arcuate nucleus, dorsomedial hypothalamic nucleus and lateral hypothalamic area were removed from the -2 and $-5 \mathrm{~mm}$ slices. Tissues were stored at $-80^{\circ} \mathrm{C}$ until analysis. For western blot analysis, brown adipose tissue was homogenised as previously described [22]. Protein was determined using the DC protein assay kit (Bio-Rad, Hercules, CA, USA).

Serum leptin, NEFA, triglyceride and cholesterol

Serum leptin was measured using a rat radioimmunoassay kit (Linco Research). Serum NEFA, triglyceride and total cholesterol levels were determined by enzymatic colorimetric kits from Wako Chemicals (Richmond, VA, USA).

\section{RT-PCR}

Expression levels of Pomc, neuropeptide Y (Npy), agoutirelated protein $($ Agrp $)$, melanocortin 3 receptor $(M c 3 r)$, melanocortin 4 receptor $(M c 4 r)$ in the hypothalamus were identified by relative quantitative RT-PCR using the QuantumRNA 18S Internal Standards kit (Ambion, Austin, TX, USA) as described previously [22, 29]. Relative quantitative PCR was performed by multiplexing corresponding primers (Pomc sense 5'-GCTTGCAAACTCGACCTCTC3', antisense 5-CTTGATGATGGCGTTCTTGA-3'; Npy sense 5'-ATGGGGCTGTGTGGACTGACC-3', antisense 5'- GTCAGGAGAGCAAGTTTCATTT-3'; Agrp sense 5'AGGGCATCAGAAGGCCTGACCA-3', antisense 5'-CT TGAAGAAGCGGCAGTAGCAC-3'; $M c 3 r$ sense 5'-AG CAACCGGAGTGGCAGT-3'; antisense 5'-GGCCACGA TCAAGGAGAG-3'; $M c 4 r$ sense 5'-AGTCTCTGGGGA AGGGGCA-3'; antisense 5'-CAACTGATGATGATCCCG AC-3'), 18S primers and competimers, and coamplifying. The linearity for all amplicons was determined to be between 20 and 29 cycles. The optimum ratio of $18 \mathrm{~S}$ primer to competimer was 1:5 for Pomc, 1:7 for Npy, 1:4 for Agrp, 1:6 for $M c 3 r$ and 1:9 for $M c 4 r$. PCR was performed at $94^{\circ} \mathrm{C}$ denaturation for $60 \mathrm{~s}, 59^{\circ} \mathrm{C}$ annealing temperature for $50 \mathrm{~s}$, and $72^{\circ} \mathrm{C}$ elongation temperature for $50 \mathrm{~s}$ for 26 (Pomc), 22 (Npy), 25 (Agrp), 23 (Mc3r) or 27 (Mc4r) cycles. The PCR product was electrophoresed on acrylamide gel and stained with SYBR green (Molecular Probes, Eugene, OR, USA). Gels were scanned using a Storm fluorescent scanner (Molecular Dynamics, Sunnyvale, CA, USA) and data analysed using ImageQuant (Molecular Dynamics). The relative value of each mRNA was derived by dividing the signal obtained for the corresponding amplicon by that for the $18 \mathrm{~S}$ amplicon.

\section{UCP1 protein}

Immunoreactive uncoupling protein 1 (UCP1) was determined with an antibody specific to rat UCP1 (Linco Research). Brown adipose tissue homogenates $(20 \mu \mathrm{g})$ prepared as described previously [22] were separated on an SDS-PAGE gel and electrotransferred to nitrocellulose membrane. Immunoreactivity was assessed with an antibody specific to UCP1. Immunoreactivity was visualised by enhanced chemiluminescent detection (Amersham Pharmacia Biotech, Piscataway, NJ, USA) and quantified by densitometry.

\section{Statistical analysis}

Results are presented as means $\pm \mathrm{SE}$. Repeated measures ANOVA was used for analyses of body weight and food intake. Two-way ANOVA was employed for analyses of Pomc gene expression in different areas of the hypothal- 
amus. When the main effect was significant, a post hoc test ( $t$-test or the Bonferroni method) was applied to determine individual differences between means. For all other data, unpaired two-tailed Student's $t$-test was employed. A value of $p<0.05$ was considered significant.

\section{Results}

Pomc expression in the hypothalamus of obese aged rats

To verify overexpression of the Pomc transgene following central bilateral viral delivery, Pomc mRNA was measured in the whole hypothalamus by RT-PCR (Fig. 2a). Forty-two days after vector delivery, hypothalamic Pomc mRNA lev-

a

Pomc (540 bp)

18S rRNA (315 bp)
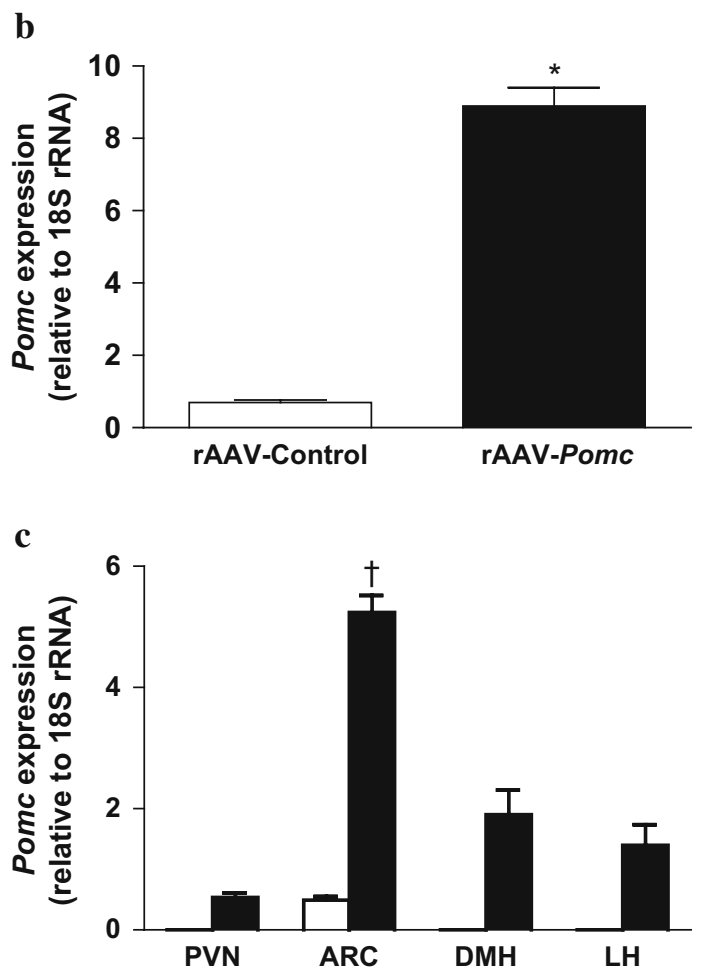

Fig. 2 Hypothalamic Pomc expression 42 days after rAAV-Pomc or control vector delivery in aged-obese rats. a Representative image of relative quantitative RT-PCR analysis of whole hypothalamic Pomc mRNA with $18 \mathrm{~S}$ rRNA as an internal standard. b Quantification of Pomc mRNA normalised to $18 \mathrm{~S}$ RNA. c Comparisons of Pomc mRNA levels in the paraventricular nucleus $(P V N)$, arcuate nucleus $(A R C)$, dorsomedial hypothalamic nucleus $(D M H)$ and lateral hypothalamic area $(L H)$ of control (open bars) and rAAV-Pomc-treated (closed bars) rats. Data are mean $\pm \mathrm{SE}$ from six rats per group. ${ }^{*} p<0.001$ vs control by unpaired $t$-test; ${ }^{\dagger} p<0.001$ vs all others by two-way ANOVA with post-hoc Bonferroni test els were elevated 12-fold in obese aged F344/BN rats given rAAV-Pomc compared with those given rAAV-control $(p<0.001)$. Using the brain punch technique, in two additional groups of rats we identified Pomc mRNA only in the arcuate nucleus of the control rats, while in rAAVPomc rats the highest levels of Pomc expression were located in the arcuate nucleus (an 11-fold increase over the counterpart in controls). In addition, a significant amount of Pomc mRNA was also found in the dorsomedial hypothalamic nucleus and lateral hypothalamic area, and the lowest levels were observed in the paraventricular nucleus (Fig. 2b).

Food intake and body weight

Bilateral delivery of rAAV-Pomc into the basomedial hypothalamus resulted in a sustained reduction in body weight and a transient (19 days) suppression of food intake in aged obese F344/BN rats (Fig. 3). Both rAAV-Pomc- and rAAVcontrol-treated rats had transient anorexia after surgical administration of vectors but completely recovered their food intake from day 5 after vector injection. Pomc gene delivery significantly reduced food consumption from day
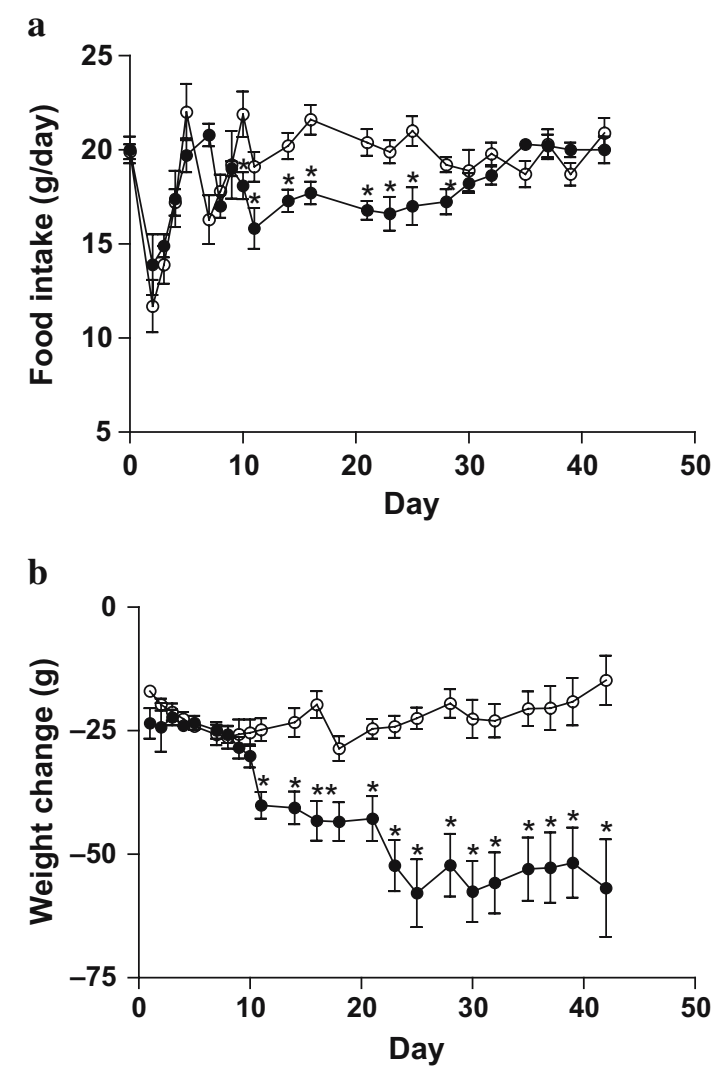

Fig. 3 Food consumption (a) and body weight change (b) after rAAV-Pomc (closed circles) or rAAV-control (open circles) delivery in aged obese rats. The vectors were injected at day 0 . Data are means \pm SE of six rats per group. $p<0.001$ for difference in food intake (a) and weight change (b) with treatment by repeated measures ANOVA. ${ }^{*} p<0.05$ for difference in food intake (a) or weight change (b) by unpaired $t$-test at the individual time point 
10 after vector injection, and these rats ate $2-4 \mathrm{~g}$ less per day compared with rAAV-control rats. However, this anorexia attenuated by day 30 (Fig. 3a). The patterns of feeding and weight change of the surgical sham control were very similar to those of rAAV-control rats during the whole experimental period (data not shown). There was a steady decrease in body weight following Pomc gene delivery during most of the period when food consumption was diminished. Near the end of and after attenuation of the anorexic response, body weight reached a plateau. In contrast to the return of food consumption to control level, the weight reduction was sustained for the duration of the experiment (Fig. 3b). Before and on the day of vector delivery, average body weight of rAAV-Pomc-treated rats was comparable to that of rAAV-control rats $(591 \pm 23$ vs $569 \pm 18 \mathrm{~g}$ at day $0, p=0.5)$. Immediately after vector delivery, both rAAV-Pomc- and rAAV-control-treated rats lost 20-25 g of body weight. This is probably a result of surgical insult, although we cannot rule out the possibility that a viral infection in the hypothalamus may play a minor role. While body weight of rAAV-control rats remained steady throughout the whole experimental period, rats given rAAV-Pomc lost more weight starting from day 10 , and the difference in body mass between the two groups gradually increased over the 42 days (Fig. $3 b$ ). At the end of the experiment (day 42), rAAV-Pomc rats had lost an average of $9.5 \%$ of their initial body weight compared with only $2.7 \%$ in control rats (weight change $-56.8 \pm$ 9.9 vs $-14.8 \pm 5.0 \mathrm{~g}, p<0.01)$.

Visceral adiposity and serum leptin levels

Because Pomc gene therapy reduced the body weight of the aged obese rats, body adiposity levels were assessed. Fortytwo days after central Pomc gene delivery, there were significant reductions in the visceral adiposity, as reflected by a $19 \%$ reduction in the sum of the perirenal, retroperitoneal and epididymal white adipose tissues $(p<0.05)$ in rAAVPomc-treated compared with control rats (Fig. 4a). Given the difference in overall body weight, we also normalised the sum of the three regions of adipose tissues to the total body weight. By this calculation, there was also a significant reduction in visceral adiposity with Pomc gene delivery compared with controls $(4.56 \pm 0.25 \%$ of total body weight vs $5.21 \pm 0.22 \%, p<0.05)$. Serum leptin levels, one indicator of body fat mass [32], were 33\% lower in the rAAV-Pomc group compared with the control group (Fig. 4b). In rats killed on day 22 after central Pomc gene delivery, there was only a non-significant $11 \%$ reduction in visceral adiposity $(23.4 \pm 1.4 \mathrm{~g}$ vs rAAV-control $26.4 \pm 1.5 \mathrm{~g}$, $p=0.17)$ and a $27 \%$ decrease in serum leptin $(15.2 \pm 1.7 \mathrm{ng} /$ $\mathrm{ml}$ vs $\mathrm{rAAV}$-control $20.8 \pm 1.0 \mathrm{ng} / \mathrm{ml}, p<0.05)$.

Intraperitoneal glucose tolerance test

There is mounting evidence suggesting that the central melanocortin system plays a role in the regulation of glucose
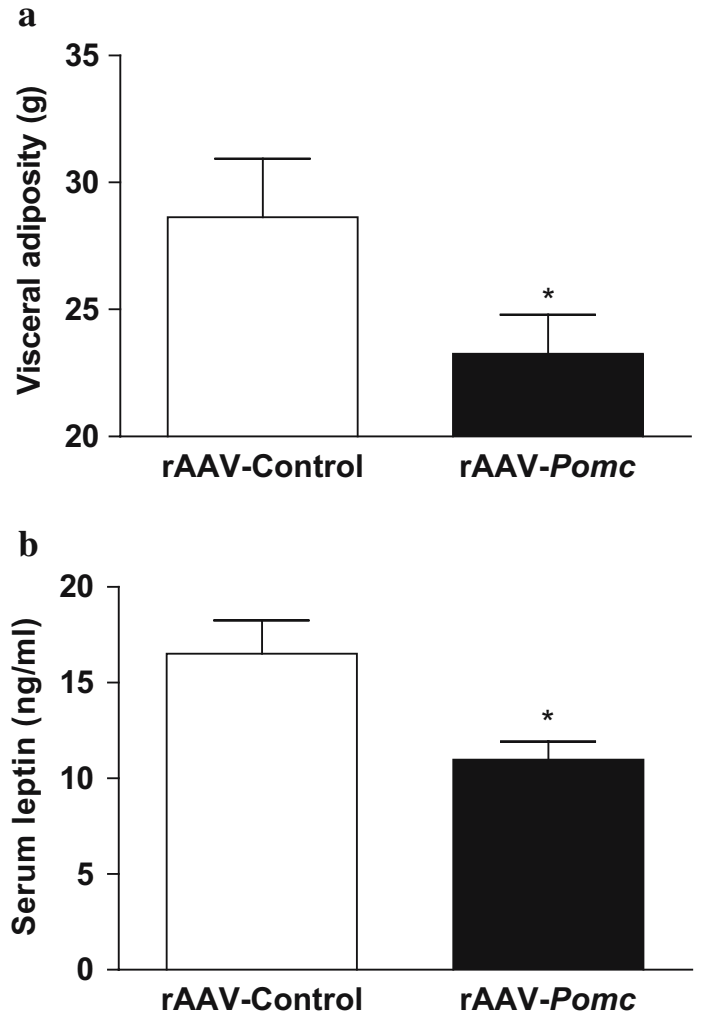

Fig. 4 Visceral adiposity (a) and fasting serum leptin (b) 42 days after rAAV-Pomc or rAAV-control delivery in aged obese rats. Visceral adiposity levels are represented by the sum of perirenal, retroperitoneal and epididymal white adipose tissues. Data are means \pm SE of six rats per group. ${ }^{*} p<0.05$ vs. control by unpaired $t$-test

metabolism. Therefore, we performed an IPGTT on days 20 and 36 after vector injection. On day 20, when the differences in body weight and adiposity were much smaller than those on day 42, Pomc gene delivery had not altered the glucose metabolism (Fig. 5a). As shown in Fig. 5b, although the fasting blood glucose levels of rAAV-control and rAAV-Pomc-treated rats were similar, Pomc gene delivery significantly accelerated glucose clearance after glucose administration on day 36 ( $p<0.05$ by repeated ANOVA). The capacity to metabolise glucose in these aged obese rats, as indicated by the area under the curve in the IPGTT, was improved by $19 \%$ following rAAV-Pomc treatment. Consistent with these findings, plasma insulin levels at each time point were reduced in rAAV-Pomc-treated rats compared with rAAV-control rats following the glucose challenge (Fig. 5c).

\section{Serum NEFA, triglyceride and cholesterol}

Forty-two days of Pomc gene delivery reduced serum NEFA and triglyceride by $30 \%$ and $15 \%$, respectively (Fig. 6a,b). Meanwhile, serum cholesterol levels were comparable between rAAV-Pomc and control groups (Fig. 6c). 
a

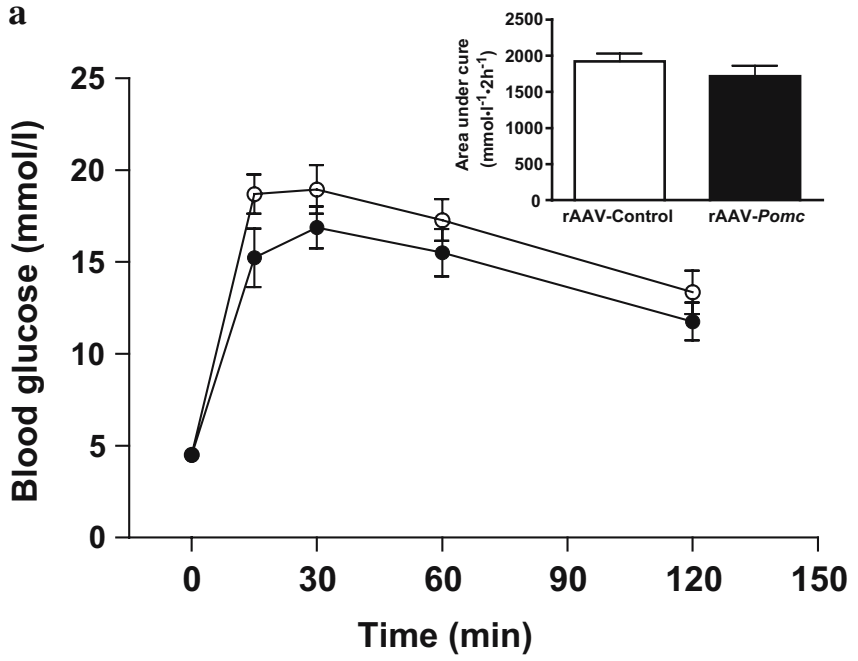

b

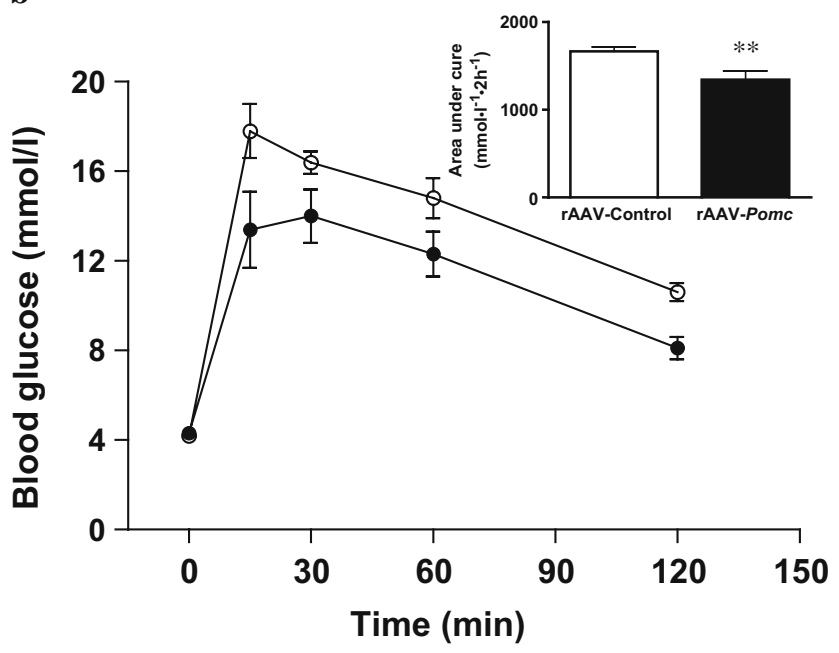

c

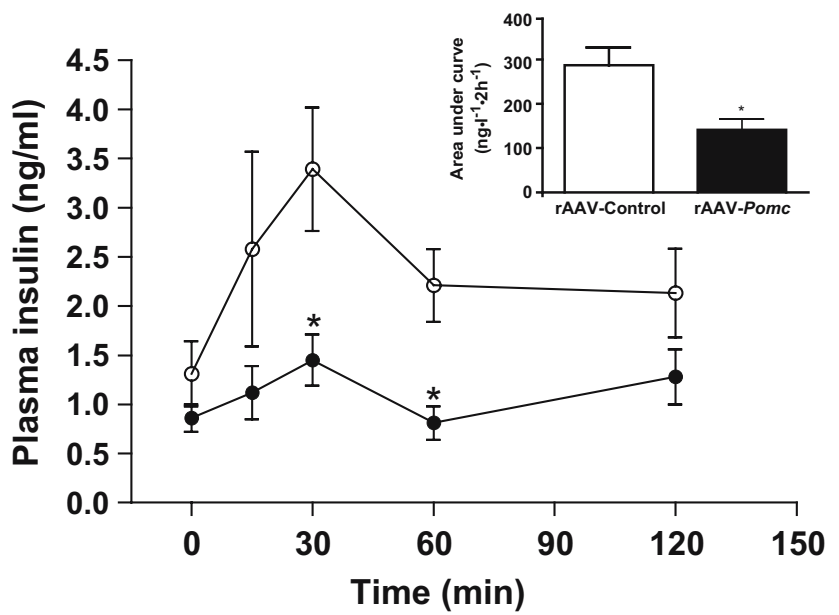

Fig. 5 Levels of blood glucose on day 20 (a) and day 36 (b) and plasma insulin on day 36 (c) after rAAV-Pomc (closed circles) or rAAV-control (open circles) delivery in aged obese rats following intraperitoneal administration of glucose $(2 \mathrm{~g} / \mathrm{kg}$ body weight; time 0 ). Data are means $\pm \mathrm{SE}$ of six rats per group. $p<0.05$ for difference in blood glucose (b) and plasma insulin (c) with treatment by repeated measures ANOVA. ${ }^{*} p<0.05 ;{ }^{* *} p<0.01$ vs control by unpaired $t$-test a

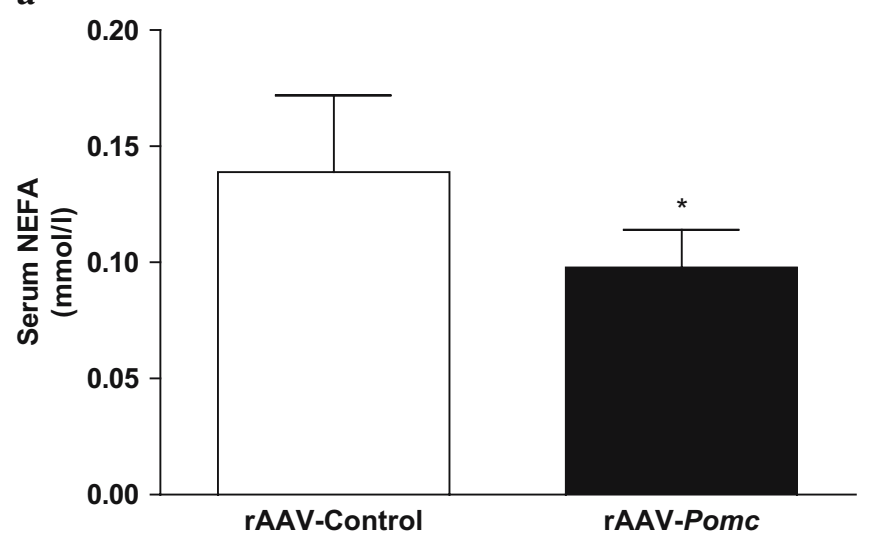

$\mathbf{b}$

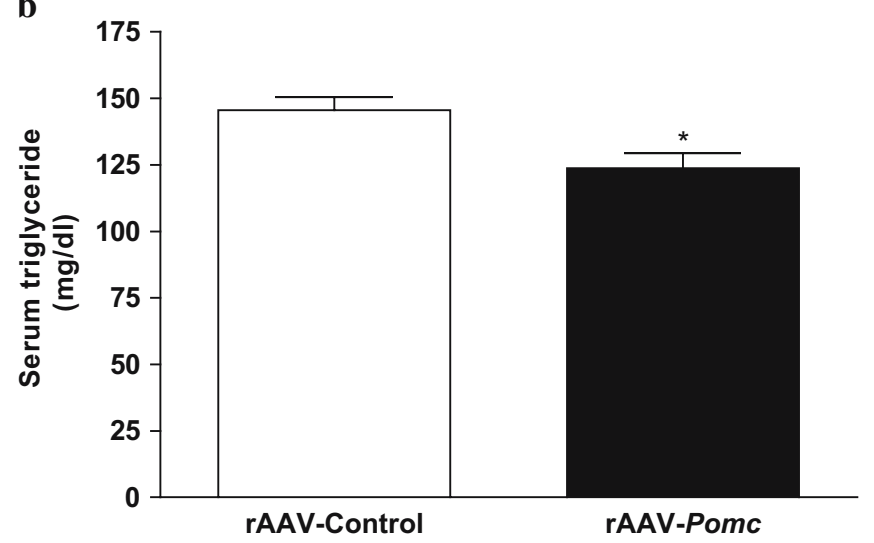

c

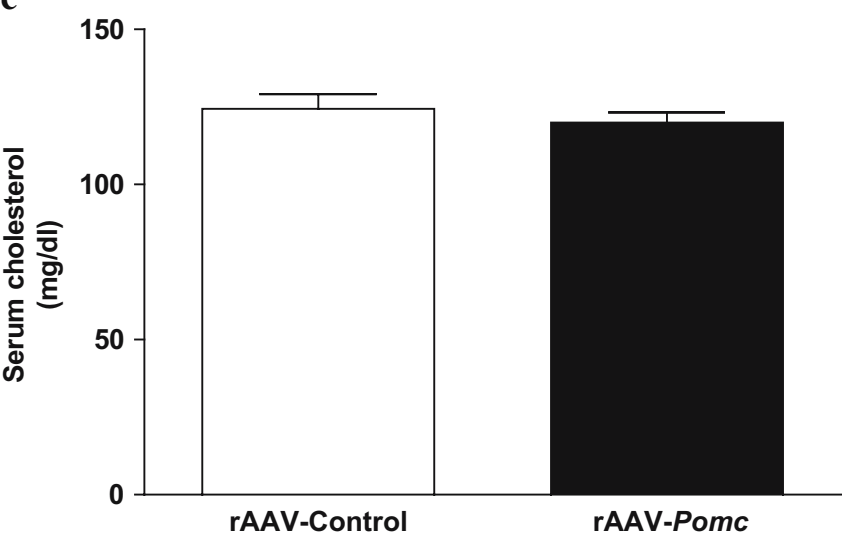

Fig. 6 Serum NEFA (a), triglyceride (b) and cholesterol (c) levels 42 days after rAAV-Pomc or rAAV-control delivery in aged obese rats. Data are mean \pm SE of six rats per group. ${ }^{*} p<0.05$ vs control by unpaired $t$-test

\section{Brown adipose tissue}

Induction of UCP1 in brown adipose tissue is an important marker for enhanced thermogenesis and thus energy expenditure in rodents $[33,34]$. In the present study, we examined the UCP1 protein levels 42 days after Pomc gene delivery. Total brown adipose tissue weight markedly declined with rAAV-Pomc treatment, whereas the protein concentration (per unit of that tissue) increased dramati- 
Table 1 Uncoupling protein 1 and brown adipose tissue parameters 42 days after rAAV-Pomc or rAAV-control delivery

\begin{tabular}{lcc}
\hline & \multicolumn{2}{l}{ Treatment } \\
\cline { 2 - 3 } & $\begin{array}{l}\text { rAAV- } \\
\text { control }\end{array}$ & $\begin{array}{l}\text { rAAV- } \\
\text { Pomc }\end{array}$ \\
\hline BAT weight (mg) & $689 \pm 28$ & $488 \pm 39^{* *}$ \\
BAT protein (mg/g BAT) & $67.3 \pm 3.5$ & $92.5 \pm 4.8^{* *}$ \\
BAT protein (mg/total BAT) & $46.2 \pm 2.8$ & $44.5 \pm 2.7$ \\
UCP1 protein (arbitrary units/g BAT) & $100 \pm 6$ & $171 \pm 22^{*}$ \\
UCP1 protein (arbitrary units/total BAT) & $100 \pm 11$ & $155 \pm 16^{*}$ \\
\hline
\end{tabular}

Data are means \pm SE of six rats per group. For UCP1 protein, the levels in rAAV-control rats were set to 100 and SE adjusted proportionally. BAT brown adipose tissue; UCP1 uncoupling protein 1

${ }^{*} p<0.05$ and ${ }^{*} p<<0.01$ vs control by unpaired $t$-test

cally, suggesting that the reduction in brown adipose tissue mass was due to the lipolysis associated with the activation of this tissue. This was supported by a $55 \%$ increase in brown adipose tissue UCP1 protein level in the rAAVPomc-treated compared with the control rats (Table 1).

Hypothalamic Npy, Agrp, Mc3r and Mc4r mRNA levels

To assess the effects of Pomc gene delivery on the expression of hypothalamic neuropeptides, Npy and Agrp mRNA levels were measured 42 days after Pomc vector delivery (Table 2). RT-PCR revealed that the expression of Agrp, the endogenous antagonist of melanocortin receptors, was unchanged in rats given rAAV-Pomc compared with control rats, whereas the mRNA levels of another potent orexigenic neuropeptide, $N p y$, tended towards a decrease $(p=0.14)$.

MC3R and MC4R are the predominant melanocortin receptors in the hypothalamus and mediate the effects of POMC-derived $\alpha$-melanocyte-stimulating hormone $(\alpha-\mathrm{MSH})$ on the homeostatic regulation of body weight.

Table 2 Hypothalamic neuropeptides, melanocortin 3 receptor $(M c 3 r)$ and melanocortin 4 receptor $(M c 4 r) 42$ days after rAAVPomc or rAAV-control delivery

\begin{tabular}{lll}
\hline & Treatment & \\
\cline { 2 - 3 } & rAAV-control & rAAV-Pomc \\
\hline Npy & $1.64 \pm 0.12$ & $1.37 \pm 0.11$ \\
Agrp & $0.52 \pm 0.05$ & $0.53 \pm 0.04$ \\
$M c 3 r$ & $0.60 \pm 0.05$ & $0.50 \pm 0.03$ \\
Mc4r & $0.77 \pm 0.06$ & $0.58 \pm 0.04^{*}$ \\
\hline
\end{tabular}

Data are means \pm SE of six rats per group

All mRNA levels were measured by relative quantitative RT-PCR with $18 \mathrm{~S}$ rRNA as internal standard. The relative value of each mRNA was derived by dividing the signal obtained for corresponding amplicon by that for $18 \mathrm{~S}$ amplicon

Npy neuropeptide Y; Agrp agouti-related protein; $M c 3 r$ melanocortin 3 receptor; $M c 4 r$ melanocortin 4 receptor $* p<0.05$ vs control by unpaired $t$-test
The delivery of rAAV-Pomc reduced the expression levels of hypothalamic $M c 3 r$ and $M c 4 r$ by $17 \%(p=0.09)$ and $25 \%(p<0.05)$, respectively, compared with rAAV-control (Table 2).

\section{Discussion}

The present study examined the long-term consequences of central rAAV-mediated Pomc gene therapy in aged obese F344/BN rats. Our findings are in agreement with our earlier short-term pharmacological study indicating that direct activation of the central melanocortin system by the $\alpha$-MSH analogue melanotan II (MTII) is effective in partially reversing the obese phenotype in aged rats [19]. Our present approach in particular resulted in long-term overexpression of Pomc in the basal hypothalamus using serotype $5 \mathrm{rAAV}$, and these aged rats responded with significant reductions in both food intake and body weight. To our knowledge, this is the first study reporting the effectiveness of gene delivery into the hypothalamus using serotype $5 \mathrm{rAAV}$. To date, eight serotypes of primate adeno-associated virus (AAV) have been identified [35]. Of these, type $2 \mathrm{AAV}$ is best characterised and most employed in gene therapy studies. Type 5 AAV has a distinct advantage over type 2 because of a higher level of transduction efficiency in certain tissues [24, 25]. Our observation of the robust expression of transgene Pomc in the hypothalamus following type 5 rAAV-Pomc delivery suggests that type $5 \mathrm{rAAV}$ is a viable option for long-term gene delivery in the hypothalamus.

The present study provides several distinct sets of salient findings. First, in aged obese rats, rAAV-mediated Pomc gene delivery results in a sustained reduction in body weight despite short-term suppression of food consumption. The decrease in food intake in rAAV-Pomctreated rats commenced at day 10 after vector injection and abated on day 30 and afterwards. Meanwhile, starting from day 10 after vector administration, rats given rAAV-Pomc consistently lost more body weight when compared with rats given rAAV-control. For the most part, the greatest decrease in body weight occurred during the period when food intake was diminished. Soon after the anorexia attenuated, the average body weight of Pomc-vector-treated rats reached a nadir and then stabilised. Interestingly, food intake returned to precisely the same levels as before treatment. A recent report [36] suggested that melanocortins primarily regulate body adiposity rather than food intake. Based on this concept, the melanocortin-induced anorexic response is adjusted as necessary to achieve a specific level of adiposity. Thus, once a certain level of adiposity concordant with the degree of melanocortin signalling is achieved, as in the present study, the anorexic response wanes. The absence of a rebound in body weight in rAAVPomc-treated animals after the attenuation of anorexia also indicates that another component of body weight homeostasis, energy expenditure, must be persistently elevated in response to central Pomc gene therapy. We previously demonstrated that an increase in energy expenditure is sufficient to prevent the regain in body weight following 
anorexia [37]. In the present study, UCP1 protein levels were significantly elevated 42 days after Pomc vector delivery and 2 weeks after the attenuation of the POMCmediated inhibition in food intake. UCP1-mediated nonshivering thermogenesis in brown adipose tissue represents an essential element in adaptive energy expenditure in rodents. An increase in UCP1 protein is indicative of increased brown adipose tissue-facilitated energy expenditure $[33,34]$. It is known that pharmacological activation of the melanocortin system augments energy expenditure in rodents. For example, normal animals treated with the $\alpha$-MSH analogue MTII have elevated levels of brown adipose tissue UCP1 expression compared with pair-fed controls [38]. In our previous study, a serotype 2 rAAVPomc vector also markedly stimulated brown adipose tissue thermogenesis in obese Zucker rats 38 days after vector delivery [22]. Moreover, transgenic MSH overexpression in lean and obese $d b / d b$ mice reduced weight gain and adiposity without affecting food intake [39]. Although lacking direct evidence of whole-body energy expenditure, we suggest that, in addition to the hypophagia, an increase in energy expenditure, such as fat oxidation within brown adipose tissue, white adipose tissue or muscle, contributed to the amelioration of body weight and fat in aged obese rats following central Pomc gene therapy and, in particular, was instrumental in maintaining the lost weight after the anorexia attenuated.

Second, although the 19-day anorexic response to Pomc gene delivery attenuated, the onset of this tachyphylaxis to central Pomc gene delivery was markedly delayed compared with attenuation of the anorexic response following pharmacological administration of $\alpha$-MSH or MTII in either normal or dietary obese mice and rats $[20,21]$. The suppression of food consumption lasted no longer than 4 days in any of these latter studies. Additionally, in our recent study, tachyphylaxis to MTII occurred within 6 days after central infusion of MTII in aged obese F344/BN rats [19]. The mechanism of the rapid attenuation to melanocortin treatment in pharmacological studies is not clear, but may involve agonist-mediated receptor internalisation [40]. In contrast to these pharmacological studies, adeno-associated virus-mediated Pomc gene therapy suppressed food intake for up to 38 days in obese Zucker rats [22] and, in the present study, for 19 days in aged obese rats. Therefore, the prolonged anorexic response we observed in aged and obese Zucker rats may be unique to central Pomc gene delivery. Considering that Pomc is expressed at only two locations in the brain-the arcuate nucleus of the hypothalamus and the nucleus of the tractus solitarius of the brainstem-we delivered Pomc vector into the basal medial hypothalamus, aiming at the arcuate nucleus, where Pomcexpressing neurones are located. With this procedure, the overproduction of $\alpha$-MSH derived from transgene Pomc expression is presumably assisted by a variety of endogenous enzymes, such as prehormone convertases, carboxypeptidase $\mathrm{E}$ and peptidyl $\alpha$-amidating mono-oxygenase [41]. This endogenously regulated production of $\alpha-\mathrm{MSH}$ may help prevent the rapid desensitisation witnessed in previous pharmacological studies. The prolonged anorexic response could also be attributed to potential overproduction of other peptides in addition to $\alpha-\mathrm{MSH}$, such as $\beta$ $\mathrm{MSH}$ and $\beta$-endorphin, both of which are normally derived from the POMC precursor. It has been suggested that these peptides also participate in the regulation of energy balance $[42,43]$. However, with our approach, the rAAV-mediated overexpression of Pomc in the hypothalamus did result in the transfection of neural cells outside the arcuate nucleus, most notably in the dorsomedial hypothalamic nucleus and lateral hypothalamic area. The potential ectopic expression of Pomc in the brain might account for some of the responses observed.

Central Pomc gene therapy also appears to affect the expression of the two important central melanocortin receptors. When assessed at the time the animals were killed, hypothalamic $M c 4 r$ expression was significantly reduced, whereas $M c 3 r$ mRNA levels tended to decrease in the rAAV-Pomc rats. Interestingly, the expression of the two orexigenic neuropeptides, Npy and Agrp, did not change after Pomc gene delivery. These data suggest that prolonged exposure to Pomc gene overexpression in aged animals may downregulate hypothalamic melanocortin receptors. This may be one desensitisation mechanism for the attenuation of anorexia evoked by Pomc gene therapy.

Lastly, rAAV-Pomc gene delivery improves glucose metabolism and insulin sensitivity in aged obese rats. Aged F344/BN rats are associated with insulin resistance and glucose intolerance, as shown in several previous reports $[15,18,44]$. In the present study, we also observed impaired glucose tolerance, as indicated by the elevated blood glucose levels after glucose loading in the aged obese F344/BN rats. Central Pomc gene therapy partially normalised glucose levels during IPGTT, and also reduced serum insulin levels markedly at all time points after glucose loading. These data are indicative of improved glucose metabolism and insulin sensitivity by Pomc gene delivery and in agreement with previous findings that central melanocortin receptor activation suppresses insulin release from the pancreas and enhances glucose metabolism [2, 6, 22, 38, 45]. Since glucose metabolism was not significantly improved on day 20 after Pomc gene delivery, when the differences in body weight reduction and visceral adiposity levels were not as large as those on day 36, it suggests that the improvement in glucose metabolism is mainly the consequence of the decreased food consumption and body weight rather than the direct result of central Pomc overexpression. In addition to its impact on insulin and glucose, Pomc gene delivery also reduced serum triglyceride and NEFA levels in the obese aged rats. Such effects could be due to the reduced lipogenesis and/or increased lipolysis in white fat tissues, along with the enhanced non-shivering thermogenesis in brown adipose tissue following Pomc gene therapy.

In conclusion, targeted Pomc gene delivery to the hypothalamus suppressed food intake, diminished body weight and reduced visceral adiposity in aged obese rats. This treatment also improved glucose and fat metabolism and insulin sensitivity. The induced hypophagia and stimulated brown adipose tissue thermogenesis are the likely mechanisms underlying these improvements, and these data suggest 
that long-term activation of the central melanocortin system may be a viable strategy to combat age-related obesity.

Acknowledgements This work was supported by the Medical Research Service of the Department of Veterans Affairs and NIH grant AG20985.

\section{References}

1. Cone RD (1999) The central melanocortin system and energy homeostasis. Trends Endocrinol Metab 10:211-216

2. Fan W, Boston BA, Kesterson RA, Hruby VJ, Cone RD (1997) Role of melanocortinergic neurons in feeding and the agouti obesity syndrome. Nature 385:165-168

3. Huszar D, Lynch CA, Fairchild-Huntress V et al (1997) Targeted disruption of the melanocortin-4 receptor results in obesity in mice. Cell 88:131-141

4. Mizuno TM, Mobbs CV (1999) Hypothalamic agouti-related protein messenger ribonucleic acid is inhibited by leptin and stimulated by fasting. Endocrinology 140:814-817

5. Butler AA, Kesterson RA, Khong K et al (2000) A unique metabolic syndrome causes obesity in the melanocortin-3 receptor-deficient mouse. Endocrinology 141:3518-3521

6. Obici S, Feng Z, Tan J, Liu L, Karkanias G, Rossetti L (2001) Central melanocortin receptors regulate insulin action. J Clin Invest 108:1079-1085

7. Mizuno TM, Kleopoulos SP, Bergen HT, Roberts JL, Priest CA, Mobbs CV (1998) Hypothalamic pro-opiomelanocortin mRNA is reduced by fasting and [corrected] in ob/ob and db/db mice, but is stimulated by leptin. Diabetes 47:294-297

8. Kim EM, O'Hare E, Grace MK, Welch CC, Billington CJ, Levine AS (2000) ARC POMC mRNA and PVN alpha-MSH are lower in obese relative to lean Zucker rats. Brain Res 862: $11-16$

9. Guan XM, Yu H, Van der Ploeg LH (1998) Evidence of altered hypothalamic pro-opiomelanocortin/neuropeptide Y mRNA expression in tubby mice. Brain Res Mol Brain Res 59:273279

10. Good DJ, Porter FD, Mahon KA, Parlow AF, Westphal H, Kirsch IR (1997) Hypogonadism and obesity in mice with a targeted deletion of the Nhlh2 gene. Nat Genet 15:397-401

11. Bergen HT, Mizuno TM, Taylor J, Mobbs CV (1998) Hyperphagia and weight gain after gold-thioglucose: relation to hypothalamic neuropeptide Y and proopiomelanocortin. Endocrinology 139:4483-4488

12. Mobbs CV, Bray GA, Atkinson RL et al (2001) Neuroendocrine and pharmacological manipulations to assess how caloric restriction increases life span. J Gerontol A Biol Sci Med Sci 56 (Spec No 1):34-44

13. Yaswen L, Diehl N, Brennan MB, Hochgeschwender U (1999) Obesity in the mouse model of pro-opiomelanocortin deficiency responds to peripheral melanocortin. Nat Med 5:1066-1070

14. Krude H, Biebermann H, Luck W, Horn R, Brabant G, Gruters A (1998) Severe early-onset obesity, adrenal insufficiency and red hair pigmentation caused by POMC mutations in humans. Nat Genet 19:155-157

15. Gabriely I, Ma XH, Yang XM et al (2002) Removal of visceral fat prevents insulin resistance and glucose intolerance of aging: an adipokine-mediated process? Diabetes 51:2951-2958

16. Scarpace PJ, Matheny M, Zhang Y et al (2002) Central leptin gene delivery evokes persistent leptin signal transduction in young and aged-obese rats but physiological responses become attenuated over time in aged-obese rats. Neuropharmacology 42:548-561

17. Li H, Matheny M, Nicolson M, Tumer N, Scarpace PJ (1997) Leptin gene expression increases with age independent of increasing adiposity in rats. Diabetes 46:2035-2039
18. Larkin LM, Reynolds TH, Supiano MA, Kahn BB, Halter JB (2001) Effect of aging and obesity on insulin responsiveness and glut-4 glucose transporter content in skeletal muscle of Fischer 344×Brown Norway rats. J Gerontol A Biol Sci Med Sci 56:B486-B492

19. Zhang Y, Matheny M, Tumer N, Scarpace PJ (2004) Agedobese rats exhibit robust responses to a melanocortin agonist and antagonist despite leptin resistance. Neurobiol Aging 25: $1349-1360$

20. McMinn JE, Wilkinson CW, Havel PJ, Woods SC, Schwartz MW (2000) Effect of intracerebroventricular alpha-MSH on food intake, adiposity, c-Fos induction, and neuropeptide expression. Am J Physiol Regul Integr Comp Physiol 279:R695R703

21. Pierroz DD, Ziotopoulou M, Ungsunan L, Moschos S, Flier JS, Mantzoros CS (2002) Effects of acute and chronic administration of the melanocortin agonist MTII in mice with diet-induced obesity. Diabetes 51:1337-1345

22. Li G, Mobbs CV, Scarpace PJ (2003) Central pro-opiomelanocortin gene delivery results in hypophagia, reduced visceral adiposity, and improved insulin sensitivity in genetically obese Zucker rats. Diabetes 52:1951-1957

23. Monahan PE, Samulski RJ (2000) AAV vectors: is clinical success on the horizon? Gene Ther 7:24-30

24. Hildinger M, Auricchio A, Gao G, Wang L, Chirmule N, Wilson JM (2001) Hybrid vectors based on adeno-associated virus serotypes 2 and 5 for muscle-directed gene transfer. J Virol 75:6199-6203

25. Zabner J, Seiler M, Walters R et al (2000) Adeno-associated virus type 5 (AAV5) but not AAV2 binds to the apical surfaces of airway epithelia and facilitates gene transfer. J Virol 74: 3852-3858

26. Uhler M, Herbert E (1983) Complete amino acid sequence of mouse pro-opiomelanocortin derived from the nucleotide sequence of pro-opiomelanocortin cDNA. J Biol Chem 258:257-261

27. Daly TM, Ohlemiller KK, Roberts MS, Vogler CA, Sands MS (2001) Prevention of systemic clinical disease in MPS VII mice following AAV-mediated neonatal gene transfer. Gene Ther 8:1291-1298

28. Loeb JE, Cordier WS, Harris ME, Weitzman MD, Hope TJ (1999) Enhanced expression of transgenes from adeno-associated virus vectors with the woodchuck hepatitis virus posttranscriptional regulatory element: implications for gene therapy. Hum Gene Ther 10:2295-2305

29. Li G, Klein RL, Matheny M, King MA, Meyer EM, Scarpace PJ (2002) Induction of uncoupling protein 1 by central interleukin-6 gene delivery is dependent on sympathetic innervation of brown adipose tissue and underlies one mechanism of body weight reduction in rats. Neuroscience 115:879-889

30. Zolotukhin S, Potter M, Zolotukhin I et al (2002) Production and purification of serotype 1,2 , and 5 recombinant adenoassociated viral vectors. Methods 28:158-167

31. Paxinos G, Watson C (1997) The rat brain in stereotaxic coordinates, 3rd edn. Academic Press, San Diego

32. Frederich RC, Hamann A, Anderson S, Lollmann B, Lowell BB, Flier JS (1995) Leptin levels reflect body lipid content in mice: evidence for diet-induced resistance to leptin action. Nat Med 1:1311-1314

33. Scarpace PJ, Matheny M, Pollock BH, Tumer N (1997) Leptin increases uncoupling protein expression and energy expenditure. Am J Physiol 273:E226-E230

34. Cannon B, Nedergaard J (2004) Brown adipose tissue: function and physiological significance. Physiol Rev 84:277-359

35. Gao GP, Alvira MR, Wang L, Calcedo R, Johnston J, Wilson JM (2002) Novel adeno-associated viruses from rhesus monkeys as vectors for human gene therapy. Proc Natl Acad Sci U S A 99:11854-11859

36. Seeley RJ, Burklow ML, Wilmer KA, Matthews CC et al (2005) The effect of the melanocortin agonist, MT-II, on the defended level of body adiposity. Endocrinology (in press) 
37. Scarpace PJ, Matheny M, Zhang Y et al (2002) Leptin-induced leptin resistance reveals separate roles for the anorexic and thermogenic responses in weight maintenance. Endocrinology 143:3026-3035

38. Cettour-Rose P, Rohner-Jeanrenaud F (2002) The leptin-like effects of 3-d peripheral administration of a melanocortin agonist are more marked in genetically obese Zucker (fa/fa) than in lean rats. Endocrinology 143:2277-2283

39. Savontaus E, Breen TL, Kim A, Yang LM, Chua SC Jr, Wardlaw SL (2004) Metabolic effects of transgenic melanocyte-stimulating hormone overexpression in lean and obese mice. Endocrinology 145:3881-3891

40. Shinyama H, Masuzaki H, Fang H, Flier JS (2003) Regulation of melanocortin-4 receptor signaling: agonist-mediated desensitization and internalization. Endocrinology 144:1301-1314

41. Pritchard LE, Turnbull AV, White A (2002) Pro-opiomelanocortin processing in the hypothalamus: impact on melanocortin signalling and obesity. J Endocrinol 172:411-421
42. Appleyard SM, Hayward M, Young JI et al (2003) A role for the endogenous opioid beta-endorphin in energy homeostasis. Endocrinology 144:1753-1760

43. Harrold JA, Widdowson PS, Williams G (2003) beta-MSH: a functional ligand that regulated energy homeostasis via hypothalamic MC4-R? Peptides 24:397-405

44. Gupta G, Cases JA, She L et al (2000) Ability of insulin to modulate hepatic glucose production in aging rats is impaired by fat accumulation. Am J Physiol Endocrinol Metab 278: E985-E991

45. Mizuno TM, Kelley KA, Pasinetti GM, Roberts JL, Mobbs CV (2003) Transgenic neuronal expression of proopiomelanocortin attenuates hyperphagic response to fasting and reverses metabolic impairments in leptin-deficient obese mice. Diabetes 52: $2675-2683$ 